\title{
Plant NLRs get by with a little help from their friends
}

\author{
Authors: Joanna M. Feehan ${ }^{1}$, Baptiste Castel ${ }^{1 \#}$, Adam Bentham², Jonathan D. G. Jones ${ }^{1^{*}}$ \\ ${ }^{1}$ The Sainsbury Laboratory, Norwich Research Park, University of East Anglia, Norwich, UK \\ 2John Innes Centre, Norwich Research Park, Norwich, UK \\ \#Current Address: Department of Biological Sciences, National University of Singapore, \\ Singapore
}

${ }^{*}$ Corresponding author

Email: jonathan.jones@tsl.ac.uk

Conflict of interest statement: None to declare

\begin{abstract}
Many plant NLR (nucleotide binding, leucine-rich repeat) immune receptors require other NLRs for their function. In pairs of chromosomally adjacent sensor/helper NLRs, the sensor typically carries an integrated domain (ID) that mimics the authentic target of a pathogen effector. The RPW8-NLR clade supports the function of many diverse plant NLRs, particularly those with a TIR N-terminal domain, in concert with a family of EP-domain containing signaling partners. The NRC clade of NLRs is required for the function of many unlinked sensor NLRs in Solanaceous plants. We evaluate recent advances in paired NLR biology in the context of the structure and possible mechanisms of the first defined plant inflammasome containing ZAR1.
\end{abstract}

\section{Introduction}

Plants have evolved both cell surface and intracellular receptors that detect pathogen molecules and activate defense mechanisms. Resistance $(R)$ genes usually encode intracellular "NLR" receptors that carry a nucleotide-binding (NB) domain and a leucine-rich repeat (LRR) domain [1]. Cell surface receptor-like kinases (RLKs) such as Arabidopsis EFR and FLS2 (which detect bacterial molecules) require the helper RLK BAK1 for function [2]. Likewise, many NLRs are now known to require other NLRs for function, and we review here recent advances in the biology of plant NLR/NLR cooperation in immune signaling.

Plant NLR proteins are classified into three ancient and diverged classes based on differences in their N-terminal domain architecture: Toll/Interleukin-1 receptor/Resistance (TIR) NLRs (TNLs), non-TIR or coiled-coil (CC) NLRs (CNLs), and RPW8-like coiled-coil domain NLRs ( $\mathrm{CC}_{\mathrm{R}}-\mathrm{NLs}$ or RNLs) [3-5]. Upon effector recognition, NLRs activate a suite of defense responses that include an influx of calcium ions, production of reactive oxygen species (ROS), and transcriptional changes. An additional hallmark of effector-triggered immunity $(E T I)$ is a type of programmed cell death (PCD) known as the Hypersensitive Response (HR) [6]. Although recent discoveries in $\mathrm{R}$ protein mechanisms for pathogen recognition have advanced the field, a significant gap still exists in our knowledge of the intervening steps between pathogen recognition and defense activation. A recent comprehensive commentary in last year's issue of COPB should be read in parallel to our review [7].

Three types of NLR/NLR cooperation can be distinguished (Fig 1A). The requirement of one NLR for another was first defined when the Arabidopsis TNL RPP2 (conferring downy mildew resistance) was cloned, revealing that two adjacent TNL genes, $R P P 2 A$ and $R P P 2 B$, are both required for resistance [8]. There are now many examples of NLR pairs, one member of which carries an integrated domain ("integrated decoy") that mimics the authentic target of a pathogen effector, while the other member of the pair acts as a helper NLR 
required for conversion of effector detection into defense activation. The Arabidopsis RPS4/RRS1 TNL/TNL-WRKY (and its paralogous pair RPS4B/RRS1B), and the rice RGA4/RGA5 CNL/CNL-HMA, provide paradigmatic examples of this architecture [9]. Additional reviews and reports provide a rich analysis of the diversity of IDs found in NLRIDs, and their likely significance as revealing targets of pathogen effectors [10-12].

Another helper NLR was revealed during a search for genes that, when silenced, compromise the function of the tobacco TNL-encoding virus resistance gene $N$. NRG1 $(\mathrm{N}-$ requirement gene) encodes an RNL that is required for $N$ function [13]. Subsequently, in Arabidopsis the NRG1-related Activated disease resistance gene 1 (ADR1) gene family was found to be required for full function of several TNLs and CNLs [14]. Both NRG1 and ADR1 carry at their N-termini a domain homologous to RPW8, originally defined as an Arabidopsis powdery mildew resistance gene encoding a protein with an $\mathrm{N}$-terminal signal anchor and several coiled coil domains, but without NB or LRR domains [15]. Despite this unorthodox structure, RPW8 requires lipase-like protein Enhanced Disease Susceptibility 1 (EDS1) for function [15]. EDS1 was originally defined as essential for the function of all TNL proteins and forms functional heterodimers with either of two related lipase-like proteins, SAG101 (senescence-associated gene 101) or PAD4 (phytoalexin deficient 4). EDS1, SAG101 and PAD4 share EDS1/PAD4 (EP) domains [16,17]. NRG1 and ADR1 are encoded by genetically redundant genes in Arabidopsis but not Nicotiana benthamiana [7]. An intriguing homology was noted between the RPW8 domain, a domain in mixed-lineage kinases (MLKLs) and the fungal HeLo/HeLo-Like (HELL) domain [18], reviewed in more detail in Jubic et al. (2019) [7].

A third example is provided by the NRC (NLR Required for Cell death) class of helper NLRs required for the function of many, but not all, Solanaceae CNLs [19]. NRCs are also CNLs and are more related to NRC-requiring NLRs than non-NRC-requiring NLRs. Intriguingly, NRCs were first reported as required for full function of the receptor-like resistance protein Cf-4 [20], and for $R x$, Pto and Mi NLR gene function. Wu et al. (2017) discovered functionally redundant NRC paralogs can display distinct specificities toward different sensor NLRs that confer immunity to oomycetes, bacteria, viruses, nematodes, and insects [19]. The helper NLR NRC4 is required for the function of several sensor NLRs, including Rpi-blb2, Mi-1.2, and R1, whereas NRC2 and NRC3 are required for the function of the NLR Prf that with the protein kinase Pto detects and responds to bacterial AvrPto and AvrPtoB. Interestingly, NRC2, NRC3, and NRC4 redundantly contribute to the immunity mediated by other sensor NLRs, including Rx, Bs2, R8, and Sw5. The NRC superclade probably emerged over 100 Mya from an NLR pair that diversified to constitute up to onehalf of the NLRs of Asterids [19].

In an additional example, the Arabidopsis RPP4 TNL gene for downy mildew resistance resides in a complex locus with multiple paralogs [21], one of which is SUPPRESSOR OF NPR1, CONSTITUTIVE 1 (SNC1), originally defined by an SNC1 allele that causes constitutive defense activation [22]. In a genetic screen to identify genes required for SNC1, three other TNL in the RPP4 haplotype were found to be redundantly required for SNC1-mediated defense, named SIDEKICK SNC1 1 (SIKIC1), SIKIC2 and SIKIC3 [23]. SIKIC2 physically associates with SNC1. Most puzzling, RPP4 in the same cluster does not require SIKICs for function. Conceivably, then, the SIKIs could evolve into NRC-like helpers of SNC1-derived sensors. This may be the tip of the iceberg; many more such examples may exist but are invisible without the appropriate genetic analysis.

\section{NLR/ NLR-ID pairs}

The Arabidopsis RRS1/RPS4 TNL pair recognizes the bacterial effectors AvrRps4 and PopP2 via an integrated WRKY transcription factor domain in RRS1-R that mimics the effector's authentic targets. How the complex activates defense upon effector recognition is unknown. Deletion of the WRKY domain results in an autoactive RRS1 allele, suggesting the WRKY domain contributes to maintaining the complex in an inactive state [24]. The WRKY domain interacts with the adjacent domain 4 . The inactive state of RRS1 is maintained by 
WRKY interactions with domain 4 prior to ligand detection. AvrRps4 disrupts WRKY-domain 4 association interactions, leading to derepression of the complex [24]. PopP2-triggered activation is less easily explained by such disruption and involves the longer $\mathrm{C}$-terminal extension of RRS1-R. Following RRS1 depression, interactions between domain 4 and the RPS4 C-terminal domain likely contribute to activation.

Recently, Guo et al. (in press) shed light on how the RRS1-R C-terminus contributes to PopP2 but not AvrRps4 responsiveness [25]. The C-terminus of RRS1-R, but not RRS1$\mathrm{S}$, is phosphorylated. Phosphorylation at Thr1214 in the WRKY domain maintains RRS1-R in its inactive state, and also inhibits PopP2 acetylation of RRS1-R. PopP2 catalyses Oacetylation of Thr1214, preventing its phosphorylation. Phosphorylation at the other sites is required for PopP2, but not AvrRps4, responsiveness, and is required for PopP2-dependent $\mathrm{C}$-terminal interaction with TIR RRS1. Effector-triggered proximity between the $\mathrm{N}$-terminal TIR domain and the RRS1-S or RRS1-R C-termini promotes depression of the complex. This effector-promoted interaction between $\mathrm{TIR}^{\mathrm{RRS} 1}$ and the RRS1 C-terminus likely relieves inhibition of TIR RPS4 by TIR RRS1. Thus, effector-triggered and phosphorylation-regulated conformational changes within RRS1 result in distinct modes of derepression of the complex by PopP2 and AvrRps4.

What is known about within-species diversity in NLR/NLR-ID architectures? An important recent study used sequence capture to investigate the "panNLRome" of Arabidopsis thaliana [26]. This revealed remarkable diversity in NLR/NLR-ID pair architecture, both in ID repertoires imported into (presumed) sensor NLRs, and also in NLR presence/absence variation.

A recent study has demonstrated the first successful engineering of an NLR-ID to expand recognition to different effector alleles [27]. The NLRs Pik-1 and Pik-2 comprise a CNL pair from rice that confers resistance to the rice blast fungus, Magnaporthe oryzae, carrying the AVR-Pik effector. Pik-1 is the sensor NLR, containing a Heavy Metal Associated (HMA) domain between the CC domain and the NB-ARC domain, and Pik-2 is the helper NLR that is required for defense signalling upon effector recognition by Pik-1 [28]. There are several alleles of the Pik resistance proteins encoded at the Pik locus (Pik*, Pikm, Pikp, Piks, Pikh, Pi1, and Pi7) as well as several alleles of the AVR-Pik effectors (AVR-PikA, C, D, $E$, and F). Pik alleles have evolved to recognise different effector alleles [29]. A study dissecting the effects of allelic diversity in Pik NLRs on AVR-Pik recognition demonstrated the Pikp pair, Pikp-1 and Pikp-2, are able to recognise only one of the AVR-Pik effectors, AVR-PikD, whereas a second pair of alleles at the Pik locus, Pikm-1 and Pikm-2, have a broader AVR recognition spectrum, and is able to recognise AVR-PikD, as well as AVR-PikA and AVR-PikE [30]. Guided by structures of the HMA domains of Pikp-1 and Pikm-1 in complex with various alleles of AVR-Pik, the authors used prior knowledge of the Pikp and Pikm alleles to engineer a mutant of the Pikp-1 HMA, designated Pikp ${ }^{\mathrm{NK}-\mathrm{KE}}$, that can bind to the same alleles of AVR-Pik as the Pikm-1 HMA [27]. However, it is still unclear how the binding of the effector to the HMA domain of Pik-1 sensor NLR is relayed to the Pik-2 helper NLR; this remains one of the major questions in paired NLR function.

\section{Non-NLR sensors and their singleton helpers}

There are many examples of NLRs guarding pathogen effector targets or host decoys [31,32], and it has been proposed that NLR-IDs evolved through the integration of guardee proteins into the NLR [33]. NLRs that act as guards are usually singleton NLRs that monitor either structural or biochemical changes in their guardee, such as detection by Arabidopsis RPS2 of the action of the cysteine protease AvrRpt2 on RIN4 [34]. These guardee or decoy proteins thus act as non-NLR sensors to relay effector perception to the NLR. The recent reports regarding ZAR1 and RPS5 demonstrate the important role that their sensors, PBL2 and PBS1 respectively, have in effector detection [35-37]. In both cases, these proteins facilitate the conformational change in the NLR that would be conferred by a sensor NLR in a sensor/helper NLR pair. Engineering of non-NLR sensors could be a promising avenue for expanding effector recognition, as a single NLR can have multiple 
guardees [5]. Consistent with this, a recent BioRxiv submission by Pottinger et al. (2020) shows that by manipulating the PBS1 cleavage site, normally acted upon by AvrPphB, to respond to the Nla protease from Turnip Mosaic virus (TuMV) in Arabidopsis, enabled RPS5-dependent responsiveness to Nla protease [38]. By engineering a PBS1 protein capable of being targeted by SMV Nla protease, the authors then produced transgenic Soybean with RPS5-mediated resistance to Soybean Mosaic Virus (SMV).

\section{The MADA motif - a conserved motif in helper CNLs}

How do paired NLRs activate signaling, and can this involve mechanisms that resemble the effector-dependent membrane association of ZAR1 [35,36]? A Mu transposition screen that created random truncations of the CC domain from the helper NLR, NRC4, revealed 29 amino acids at the $\mathrm{N}$-terminus which are sufficient to cause cell death when transiently expressed in $N$. benthamiana. Computational analysis of this domain revealed a motif, MADAxVSFxVxKLxxLLxxEx, designated the "MADA motif" that is found not only in NRCs, but also in approximately $20 \%$ of all CNLs from monocot and dicot species, ranging from CNL pairs to singletons [39]. Interestingly, the MADA motif is not usually found in NRC-dependent sensor NLRs. The authors suggest the MADA motif may degenerate in sensor NLRs over evolutionary time as these NLRs have further specialized to detect effectors and signal via helper NLRs. Consistent with this, some of the Solanaceous sensor CNLs that signal through the NRCs, such as Prf and Sw5b, have acquired additional Nterminal domains upstream of their $\mathrm{CC}$ domains that are involved in effector recognition $[40,41]$. Furthermore, the ZAR1 resistosome structure highlighted the importance of the conformational change in the CC domain during activation of the NLR (Wang 2019a. Wang $2019 b$ ). The rearrangement of the four helices of the $\mathrm{CC}$ domain (denoted $\mathrm{H} 1-\mathrm{H} 4$ ) from a four-helix bundle to a funnel-like structure, likely important for membrane insertion, is facilitated by the release of the $\mathrm{H} 1$ helix from the four-helix bundle to form homotypic interactions with the $\mathrm{H} 1$ helix of several ZAR1 molecules. The $\mathrm{N}$-terminal domains found in NLRs like Prf and Sw5b would likely prevent the $\mathrm{H} 1$ helix release from the $\mathrm{CC}$ domain that occurs upon activation of ZAR1 and would sterically hinder any association between the CC domain and cell membranes. This strongly suggests the CC domains of these NLRs have moved away from a signaling function to a purely sensor function. However, amino-acids 117 of Rx can confer a similar HR phenotype, even though they lack the MADA motif, so (perhaps unsurprisingly), multiple tribes of NLRs may carry $\mathrm{N}$ termini that when oligomerized activate HR.

Puzzlingly, no interactions between NRC helpers and sensors have yet been reported. Conceivably, interactions between sensor CNLs and NRCs in Solanaceae involve yet-to-be-determined partner(s), which play a role similar to the role of the EDS1/SAG101/PAD4 complex that bridges signaling between TNLs and RNLs.

Intriguingly, ZAR1 also has a MADA motif at the N-terminus. In a key experiment, Adachi et al. (2019) swapped the MADA motifs between NRC4 and ZAR1 and showed retention of cell death signaling for either NLR, demonstrating the functional conservation of the MADA motif [39]. This observation implies the signaling mechanism of the MADA motif, and CC domain as a whole, is conserved between ZAR1 and NRC4, even though ZAR1 is a singleton NLR and the NRC is a specialized helper NLR. Interestingly, the MADA motif has not been identified in RNLs, which begs the question whether the cell death triggered by MADA-CNLs and RNLs is mediated in the same way, or whether these cell death mechanisms are functionally distinct.

Whether the RNLs are required downstream of NRCs is still unclear. The potato virus $X(P V X)$ resistance protein, $R x$, was reported to require NRC helpers to trigger cell death upon detection of PVX coat protein (CP) [19]. Although dependence of Rx-mediated defense responses on RNLs has not been tested, Collier et al. (2011) demonstrated that Rx2, a close homolog of Rx, requires either NRG1 or ADR1 downstream to provide resistance to PVX. $\mathrm{R} \times 2$ would be expected to also require the NRCs to signal. This would then place RNLs 
downstream of NRCs, rather than in a parallel helper network, but this has yet to be fully resolved (Fig 1B).

\section{RNL proteins help reveal how helpers help}

ADR1 and NRG1 are RNLs, and their RPW8-like N-terminal domains are sufficient to trigger cell death [3]. They both carry a glycine zipper motif that is conserved in the fungal HET-S/HELLP proteins and the mammalian necroptosis protein MLKL $[18,42,43]$, suggesting that they all belong to the same HeLo/HELL domain category (pfam PF14479). HET-S/HELLP and MLKL proteins form amyloid aggregates and perforate the membrane to cause cell death in fungi and humans, respectively [18]. The C-terminal region of RPW8 contains repeats that determine autoimmune incompatible interactions with alleles of the NLR RPP7 [44]. This could define a novel type of prion-forming domain, with HeLo-like domains of RPW8 and RNLs initiating prisonization of RPW8. Structural information about RPW8 and an RNL N-terminus, though not available to date, would shed light on this question. The resemblance between RPW8 and the RPW8 domain of RNLs suggests that RPW8 might be involved in RNL signaling, however, an Arabidopsis rpw8 mutant does not phenocopy an $n r g 1$ mutant [45]. Furthermore, RNL-dependent NLRs such as RRS1/RPS4, RPS2 and WRR4A do not require RPW8 to signal [45]. Nevertheless, the rpw8 mutant is slightly impaired in resistance to adapted and non-adapted strains of powdery mildew, and to Pseudomonas syringae pv tomato strain DC3000 [45]. Thus, RPW8 likely plays a role in plant immunity distinct from RNL helpers.

A recent study submitted to BioRxiv functionally evaluated a family of MLKL proteins in Arabidopsis. The N-terminal HeLo domains of these AtMLKLs have been suggested to capable of inducing cell death, and also share homology with the $\mathrm{N}$-terminal domains of human MLKL and RPW8/RNL proteins [43]. Structure determination of AtMLKL1 shows an auto-repressed tetrameric complex with the HeLo domains bound to the brace domains, burying them in the structure and preventing activation. Further pursuits of the structure of an active AtMLKL complex could significantly expand our understanding of role of HeLo domains in signaling, and further inform signaling of RPW8 RNL proteins. The Arabidopsis MLKL proteins contribute quantitatively to disease resistance.

RNLs usually display low copy number and high conservation in plant genomes [46] and pan-genomes [26], consistent with a helper, rather than sensor, function [7,47]. RNLs have expanded in some plant families, including strawberry, grapevine and lettuce [48]. Conceivably, each subclade may have specialized downstream of a specific set of sensors, or alternatively, some strawberry, grapevine and/or lettuce RNLs have evolved a sensor function. Conversely, RNLs are absent in several different aquatic plant families, which correlates with absence of TNLs and the lipase-like EDS1, SAG101 and PAD4 proteins [49]. Furthermore, there is a strong indication of a co-evolved functional relationship specifically between TNLs, NRG1, and SAG101, as all three are absent from monocotyledon, and certain dicotyledon, genomes $[3,17,49]$. This supports the hypothesis that RNLs play a role in EDS1/SAG101/PAD4 signaling downstream of TNL activation [50].

An Arabidopsis "helperless" mutant that lacks all NRG1 and ADR1 genes phenocopies eds1 and pad4/sag101 mutants [47,51]. These data are consistent with a model in which RNLs and EP-domain containing proteins act in coevolved modules that requires all components for functionality (Fig 1B). Furthermore, Qi et al (2018) showed that while overexpression of NbNRG1 induces HR in an $N$. benthamiana eds1 mutant, native expression and coexpression with $\mathrm{XopQ}$ did not induce HR in this background, indicating a genetic dependence on EDS1 for NRG1 function [52]. Additionally, the autoactive ADR1${ }^{2} 2^{\mathrm{D} 484 \mathrm{~V}}$ requires PAD4 for function, and can function independently of NRG1 [47]. However, EDS1, SAG101, or PAD4 from Arabidopsis fail to complement corresponding Solanaceae mutants, supporting the hypothesis these EP-domain containing proteins have co-evolved with additional components in different plant families [53].

RNL and EP proteins likely form at least 2 distinct signaling modules that function together in immune signaling pathways downstream of TNL activation. In Arabidopsis, 
NRG1/EDS1/SAG101 is required for cell death, and ADR1/EDS1/PAD4 mediate bacterial growth restriction (Fig 1B) [50]. Interestingly, Arabidopsis alleles of NRG1/EDS1/SAG101 were sufficient for both cell death and bacterial growth restriction when expressed in $N$. benthamiana, while ADR1/EDS1/PAD4 were not required [50]. Future studies should investigate why RNLs appear to have alternate functions in different contexts.

Although there is a functional connection between NRG1 and SAG101, in Arabidopsis, NRG1 is localized to the endomembrane network [47,50] and SAG101 is primarily nuclear-localized as a dimer with EDS1 [16,17]. In N. benthamiana, however, the functional SAG101b allele is more evenly distributed between nucleus and cytoplasm [53]. Along with these differences in protein distribution, the data are ambiguous on whether direct protein interactions occur; NbEDS1 constitutively associates with NbNRG1 in transient assays in $N$. benthamiana [52], while the association between AtEDS1 and AtNRG1 alleles has not been conclusively shown $[47,50]$. There are no reports on the subcellular localization of ADR1, nor potential protein-protein associations with EDS1 or PAD4, each of which can localize in both the nuclear and cytoplasmic spaces [16]. This area of investigation is of high interest for follow-up work.

Interestingly, Lapin et al (2019) observed that if one RNL/EP module is disabled, the other likely compensates in TNL resistance, as sag101 and nrg1 mutants still show a "small but measurable portion of RRS1/RPS4/EDS1-dependent cell death" and pad4 and adr1 mutants show some resistance to bacteria carrying AvrRps4 [50]. Indeed, Saile et al. (2020) also reported the ability of NRG1 to "partially substitute for the loss of ADR1", as well as full complementation by ADR1 for NRG1 in resistances mediated by several sensor TNLs [51]. Furthermore, although isochorismate synthase (ICS1) was fully induced in an nrg1 mutant [54], NRG1s and ADR1s may still both contribute to transcriptional regulation of some genes, including SA, pipecolic acid, ROS- and HR- related genes. Whichever sensor accomplishes recognition, RNLs always regulate transcription of the same set of genes. Also, this set of RNL-regulated genes mostly overlaps with the CNL-regulated genes (with quantitative differences) [51].

However, there are discrepancies that need to be resolved; NRG1 is not required for TNL-dependent restriction of bacterial growth in Arabidopsis [47,50,54], while it is required in $N$. benthamiana [50,52]. However, overexpression of $N b N R G 1$ in Arabidopsis does restrict bacterial growth [55]. This is paradoxical given the requirement for genetically compatible alleles of NRG1/SAG101/EDS1 for cell death [50]. Furthermore, RNLs are mainly required for TNLs, but the CNL response can also be delayed or slightly reduced in a helperless mutant [51]. In addition, Schultink et al. (2017) showed TNL-mediated recognition of XopQ in Beta vulgaris [56], which does not seem to encode SAG101 or NRG1 orthologs [50]. Since most CNLs can signal independently of EP proteins, there might be also a function of RNLs independent of EP proteins.

The TIR-domains of TNLs have been shown to have NADase activity $[57,58]$ leading to production of a variant-cyclic-ADP-ribose (vcADPR) that is required for immune response. TNL proteins with mutations in the TIR domain NADase active site lose function. However, an indistinguishable compound is made by bacterial TIR proteins, but its production in plants is insufficient for immune activation [59]. This would indicate that some additional component specific to plant TIRs and EDS1 is required for immune activation. Conceivably, this involves association between the activated TNL and the RNL/EP protein module.

\section{Open Questions}

Plant NLR biology continues to perplex and fascinate. The most spectacular advance this year $[35,36]$ addresses a slightly orthogonal question to that posed in this review; why do some NLRs not appear to require helper NLRs? The structural definition of the ZAR1 resistosome may be sufficient to explain defense activation by the NLR, if membrane association and consequent ion leakage is sufficient to activate defense. The definition of the shared "MADA" motif in many NLRs, and its correlation with lack of or reduced requirement for helper NLRs, is consistent with this interpretation. As Jubic et al (2019) 
speculate, the RNL $\mathrm{N}$ terminus and MLKL family proteins may assume a similar activated structure to ZAR1, and also activate defense by modulating membrane permeability [7].

However, the mechanism(s) which helper NLRs promote responsiveness upon recognition by sensor NLRs remains enigmatic. For RPS4 and RRS1, or Pik-1/Pik-2 and RGA4/5, the sensor associates with the helper. There are few indications of the RNL associating with corresponding sensors in an effector-dependent manner. Similarly, there are not clear data on whether the NRC-dependent sensors are provoked by effectors to interact with their NRC helpers.

As these different modes of helper-dependent sensor activation become better defined, there is still scope for more systematic cross-referencing of their function. For example, the RGA4/5 pair responds to recognized effectors with an HR after transient assays in $N$. benthamiana. Is this phenotype retained in an ADR1, NRG1 mutant or an NRC2, 3, 4 triple mutant in N. benthamiana? Do NRC-dependent sensor NLRs require ADR1 and NRG1, or does the NRC pathway not require RNLs? And what contribution do ADR1 helpers make to immunity in monocots?

The cell biology of RNLs and their respective EP proteins requires further definition; there is a strong genetic link between ADR1/EDS1/PAD4 and NRG1/EDS1/SAG101, but it is not entirely clear if they physically interact to mediate function. Furthermore, it will be interesting to investigate further whether NRG1 and ADR1 interactions are involved in immune function, or whether the NRG1/EP protein signaling module is independent of the ADR1/EP protein signaling module. NRG1 appears to associate with the endomembrane system but no such information is available for ADR1. EDS1/SAG101 heterodimers are primarily localized to the nucleus while the EDS1/PAD4 heterodimers are nucleo-cytosolic. Conceivably, either NRG1 or the EDS1/SAG101 heterodimer re-localizes to mediate immune function. However, Wu et al. (2019) investigated NRG1 localization in Arabidopsis pre- and post-immune activation and found no changes [47]. Further investigation into localizations and associations of RNLs and EP proteins pre- and post-immune activation could provide insights into the mechanisms by which these modules carry out their immune functions. However, as RNL/EP modules can compensate one another (Fig 1B) and function differently given the genetic background, potentially the differences we see in Arabidopsis and $N$. benthamiana reflect different snapshots in the evolution and subfunctionalization of these modules. Comparisons between different systems may shed further light on core mechanisms of these proteins. Additionally, data from Saile et al (2020) suggests that the ADR1 part of the RNL signaling mechanisms plays a particular role in transcriptional induction, and it will be interesting understand this mechanism better.

In summary, the discovery of various kinds of helper NLR roles has greatly enriched our understanding of NLR mechanisms, but many important questions remain that are of crucial interest to all who care about eukaryotic innate immunity. 


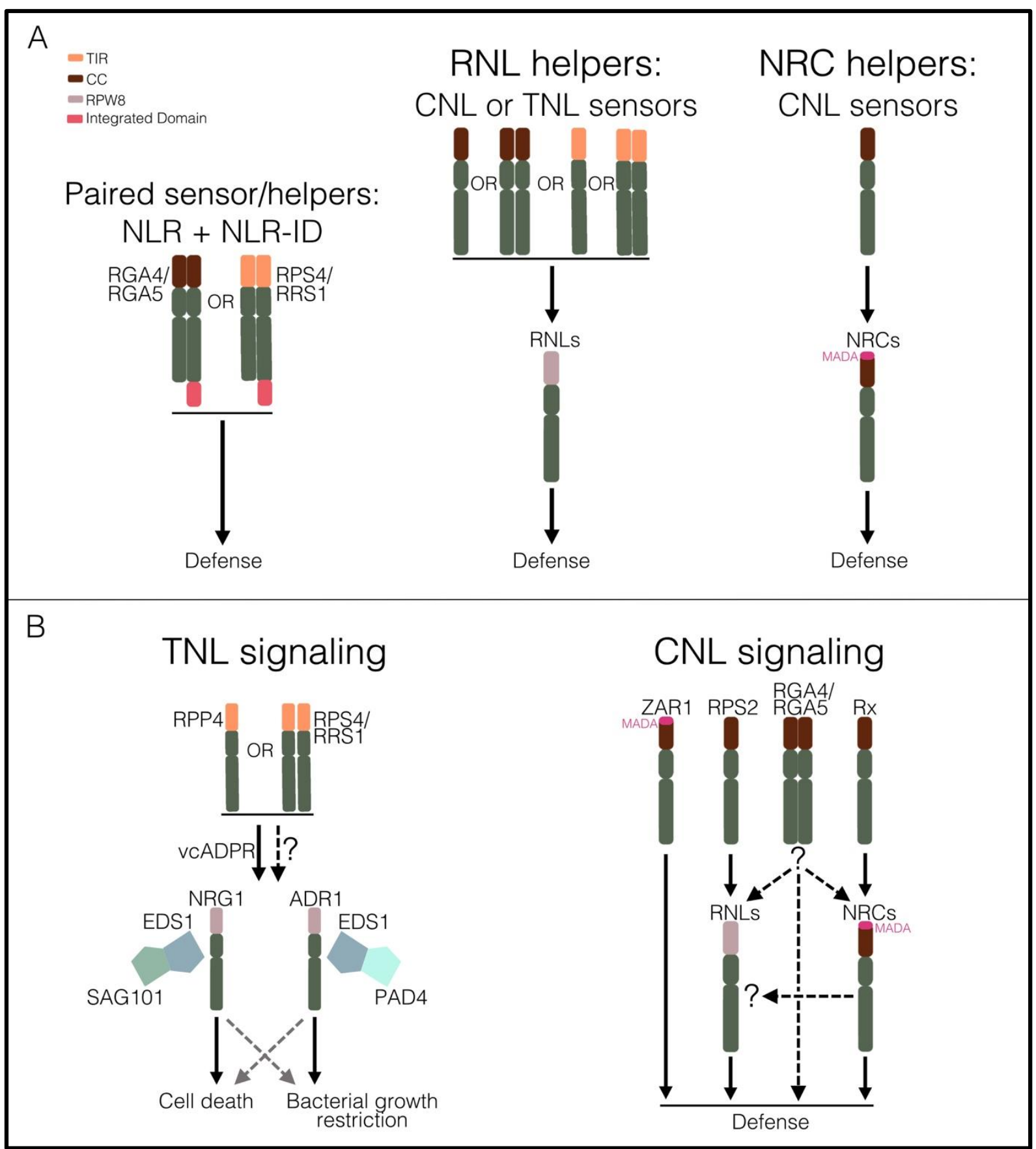

Figure 1: NLR signaling pathways. (A) Three categories of helper/sensor combination. Left; classic NLR/NLR-ID (integrated domain) pairs. Effectors interact with the ID, imposing changes that result in a conformational change within the NLR-ID that activates the helper NLR. The helper NLR may or may not need an additional helper NLR protein to signal. Centre; signaling via the RNL class of helper NLRs (NRG1 or ADR1). CNL or TNL singletons or pairs can activate RNL-dependent signaling. Right; NRC helper signaling. Particularly in Solanaceae, but also in other taxa, many (but not all) CNL sensor NLRs require NRC helper NLRs to activate defense. NRC functionality likely requires a conserved MADA domain at the $\mathrm{N}$-terminus. (B) TNL and CNL signaling pathways. Singleton or paired TNLs produce variant cyclic-ADP-Ribose (vcADPR) through NADase activity of the N-terminal TIR domains. vcADPR, in addition to unknown components, signals to RNL/EP modules of either NRG1/EDS1/SAG101 to trigger cell death, or ADR1/EDS1/PAD4 for bacterial growth restriction. Grey dotted arrows indicate the ability of these modules to mediate other defense responses either by compensation or species-specific specializations. CNLs signal either independently of helper NLRs (e.g., ZAR1), through RNLs (e.g., RPS2), or through the 
Solanaceous NRCs (e.g., Rx). It is not known how paired the CNLs RGA4/RGA5 signal downstream in defense. It is not known whether NRCs signal through RNLs to mediate defense. 
${ }^{* *}$ ADACHI, H., CONTRERAS, M. P., HARANT, A., WU, C. H., DEREVNINA, L., SAKAI, T., DUGGAN, C., MORATTO, E., BOZKURT, T. O., MAQBOOL, A., WIN, J. \& KAMOUN, S. 2019. An N-terminal motif in NLR immune receptors is functionally conserved across distantly related plant species. Elife, 8.

This paper identifies a critical motif that is essential to the signaling of cell death by CC domains from among a large number of CNLs. The authors identify this motif is overrepresented in the Solanaceous helper NLRs, the NRCs, as well as many singleton NLRs which have previously been shown to trigger cell death through their $\mathrm{N}$-terminus.

* CASTEL, B., NGOU, P. M., CEVIK, V., REDKAR, A., KIM, D. S., YANG, Y., DING, P. \& JONES, J. D. G. 2019a. Diverse NLR immune receptors activate defence via the RPW8-NLR NRG1. New Phytol, 222, 966-980.

Castel et al. confirm the requirement for NRG1 downstream of multiple TNLs and characterize the requirement for ADR1 downstream of multiple CNLs. They also reveal the ability for TNLs to signal through NRG1 or ADR1.

** DE LA CONCEPCION, J. C., FRANCESCHETTI, M., MACLEAN, D., TERAUCHI, R., KAMOUN, S. \& BANFIELD, M. J. 2019. Protein engineering expands the effector recognition profile of a rice NLR immune receptor. Elife, 8.

This paper builds upon several structural studies of M. oryzae effector and NLR-HMA domain interactions. Through structure, function, and biophysical analyses, the authors demonstrate how the HMA domain of the Pikp-1 sensor NLR can be engineered to recognise several effector alleles that are not recognised by the wildtype sensor.

* DUXBURY, Z., WANG, S., MACKENZIE, C. I., TENTHOREY, J. L., ZHANG, X., UN HUH, S., HU, L., HILL, L., NGOU, P. K., DING, P., CHEN, J., MA, Y., GUO, H., CASTEL, B., MOSCHOU, P. N., BERNOUX, M., DODDS, P. N., VANCE, R. E. \& JONES, J. D. G. submitted. Induced proximity of a TIR signaling domain on a plant-mammalian 3 NLR chimera activates defense in plants. PNAS.

The authors show the mammalian NLRC4/NAIP inflammasome can function in plants to activate induced-proximity dependent defense if a plant TIR domain is attached to NLRC4. This requires the catalytic glutamate for NADase activity. However, a bacterial TIR domain with NADase activity is insufficient for defense activation.

* GUO, H., AHN, H. K., SKLENAR, J., HUANG, J., MA, Y., DING, P., MENKE, F. L. H. \& JONES, J. D. G. in press. Phosphorylation-regulated activation of the Arabidopsis RRS1-R/RPS4 immune receptor complex reveals two distinct effector recognition mechanisms. Cell Host Microbe.

Guo et al show that the Arabidopsis RPS4/RRS1-R TNL gene pair requires C- terminal phosphorylation for PopP2 but not AvrRps4 recognition, and that interactions between RRS1 TIR domain and the WRKY integrated domain are required for depression of the complex and defense activation

* HORSEFIELD, S., BURDETT, H., ZHANG, X., MANIK, M. K., SHI, Y., CHEN, J., QI, T., GILLEY, J., LAI, J. S., RANK, M. X., CASEY, L. W., GU, W., ERICSSON, D. J., FOLEY, G., HUGHES, R. O., BOSANAC, T., VON ITZSTEIN, M., RATHJEN, J. P., NANSON, J. D., BODEN, M., DRY, I. B., WILLIAMS, S. J., STASKAWICZ, B. J., COLEMAN, M. P., VE, T., DODDS, P. N. \& KOBE, B. 2019. NAD(+) cleavage activity by animal and plant TIR domains in cell death pathways. Science, 365, 793-799. This paper, and an accompanying paper by WAN et al (2019), show that the TIR domain of plant TNL proteins has NADase activity that is required for functionality, and gives rise a variant cyclic ADPribose ( $\mathrm{V}$-cADPR) that is indistinguishable from that made by bacterial TIR domains. 
* JUBIC, L. M., SAILE, S., FURZER, O. J., EL KASMI, F. \& DANGL, J. L. 2019. Help wanted: helper NLRs and plant immune responses. Curr Opin Plant Biol, 50, 82-94.

This excellent review from last year's Biotic Interactions COPB provides much useful commentary, not duplicated here, and should be read in parallel with this review.

${ }^{* *}$ LAPIN, D., KOVACOVA, V., SUN, X., DONGUS, J. A., BHANDARI, D. D., VON BORN, P., BAUTOR, J., GUARNERI, N., RZEMIENIEWSKI, J., STUTTMANN, J., BEYER, A. \& PARKER, J. E. 2019. A coevolved EDS1-SAG101-NRG1 module mediates cell death signaling by TIR-domain immune receptors. Plant Cell.

This paper establishes the presence of distinct RNL/EP-domain containing protein modules: NRG1/EDS1/SAG101 in cell death in Arabidopsis, both cell death and bacterial growth restriction in $N$. benthamiana, and ADR1/EDS1/PAD4 in bacterial growth restriction in Arabidopsis.

* SAILE, S. C., JACOB, P., CASTEL, D., JUBIC, L. M., GONZALEZ, I. S., BÄCKER, M., JONES, J. D. G., DANGL, J. L. \& EL KASMI, F. in review.

This paper examined the phenotypes of an RNL helperless mutant and found that ADR1 and NRG1 act redundantly in ETI immune responses, defense gene regulation, and "basal resistance". They confirm that RNLs are required for TNL-mediated immune responses, but add to the literature that RNLs can "sustain" CNL-mediated immune responses also.

* WAN, L., ESSUMAN, K., ANDERSON, R. G., SASAKI, Y., MONTEIRO, F., CHUNG, E. H., OSBORNE NISHIMURA, E., DIANTONIO, A., MILBRANDT, J., DANGL, J. L. \& NISHIMURA, M. T. 2019. TIR domains of plant immune receptors are NAD(+)cleaving enzymes that promote cell death. Science, 365, 799-803.

This paper, and an accompanying paper by HORSEFIELD et al (2019), show that the TIR domain of plant TNL proteins has NADase activity that is required for functionality, and gives rise a variant cyclic ADPribose ( $\mathrm{V}$-cADPR) that is indistinguishable from that made by bacterial TIR domains.

** WANG, J., HU, M., WANG, J., QI, J., HAN, Z., WANG, G., QI, Y., WANG, H. W., ZHOU, J. M. \& CHAI, J. 2019a. Reconstitution and structure of a plant NLR resistosome conferring immunity. Science, 364.

Wang et al ( $a$ and $b)$ report the first structure of a complete plant NLR in both pre- and post activation state, and provide a paradigm-creating mechanistic insight into how recognition might drive membrane association and permeabilization to activate defense

**WANG, J., WANG, J., HU, M., WU, S., QI, J., WANG, G., HAN, Z., QI, Y., GAO, N., WANG, H. W., ZHOU, J. M. \& CHAI, J. 2019b. Ligand-triggered allosteric ADP release primes a plant NLR complex. Science, 364.

Wang et al ( $a$ and $b)$ report the first structure of a complete plant NLR in both pre- and post activation state, and provide a paradigm-creating mechanistic insight into how recognition might drive membrane association and permeabilization to activate defense

* WU, Z., LI, M., DONG, O. X., XIA, S., LIANG, W., BAO, Y., WASTENEYS, G. \& LI, X. 2019. Differential regulation of TNL-mediated immune signaling by redundant helper CNLs. New Phytol, 222, 938-953.

Wu et al. report the first evidence of a synergistic effect of NRG1 and ADR1 and reveal the genetic redundancy of these RNLs. They show that NRG1 does not relocalize away from the cytosolic space upon immune activation. 
1. Jones JD, Dangl JL: The plant immune system. Nature 2006, 444:323-329.

2. Boutrot F, Zipfel C: Function, Discovery, and Exploitation of Plant Pattern Recognition Receptors for Broad-Spectrum Disease Resistance. Annu Rev Phytopathol 2017, 55:257-286.

3. Collier SM, Hamel LP, Moffett P: Cell death mediated by the $\mathrm{N}$-terminal domains of a unique and highly conserved class of NB-LRR protein. Mol Plant Microbe Interact 2011, 24:918-931.

4. Shao ZQ, Zhang YM, Hang YY, Xue JY, Zhou GC, Wu P, Wu XY, Wu XZ, Wang Q, Wang $B$, et al.: Long-term evolution of nucleotide-binding site-leucine-rich repeat genes: understanding gained from and beyond the legume family. Plant Physiol 2014, 166:217-234.

5. Cesari S: Multiple strategies for pathogen perception by plant immune receptors. New Phytol 2018, 219:17-24.

6. Hammond-Kosack KE, Jones JD: Resistance gene-dependent plant defense responses. The Plant Cell 1996, 8:1773-1791.

7. Jubic LM, Saile S, Furzer OJ, El Kasmi F, Dangl JL: Help wanted: helper NLRs and plant immune responses. Curr Opin Plant Biol 2019, 50:82-94.

8. Sinapidou E, Williams K, Nott L, Bahkt S, Tor M, Crute I, Bittner-Eddy P, Beynon J: Two TIR:NB:LRR genes are required to specify resistance to Peronospora parasitica isolate Cala2 in Arabidopsis. Plant J 2004, 38:898-909.

9. Cesari S, Kanzaki H, Fujiwara T, Bernoux M, Chalvon V, Kawano Y, Shimamoto K, Dodds P, Terauchi R, Kroj T: The NB-LRR proteins RGA4 and RGA5 interact functionally and physically to confer disease resistance. EMBO J 2014, 33:1941-1959.

10. Sarris PF, Cevik V, Dagdas G, Jones JDG, Krasileva KV: Comparative analysis of plant immune receptor architectures uncovers host proteins likely targeted by pathogens. BMC Biology 2016, 14:8.

11. Baggs E, Dagdas G, Krasileva KV: NLR diversity, helpers and integrated domains: making sense of the NLR IDentity. Curr Opin Plant Biol 2017, 38:59-67.

12. Bailey PC, Schudoma C, Jackson W, Baggs E, Dagdas G, Haerty W, Moscou M, Krasileva KV: Dominant integration locus drives continuous diversification of plant immune receptors with exogenous domain fusions. Genome Biol 2018, 19:23.

13. Peart JR, Mestre P, Lu R, Malcuit I, Baulcombe DC: NRG1, a CC-NB-LRR protein, together with N, a TIR-NB-LRR protein, mediates resistance against tobacco mosaic virus. Curr Biol 2005, 15:968-973.

14. Bonardi V, Tang S, Stallmann A, Roberts M, Cherkis K, Dangl JL: Expanded functions for a family of plant intracellular immune receptors beyond specific recognition of pathogen effectors. Proc Natl Acad Sci U S A 2011, 108:16463-16468.

15. Xiao S, Ellwood S, Calis O, Patrick E, Li T, Coleman M, Turner JG: Broad-spectrum mildew resistance in Arabidopsis thaliana mediated by RPW8. Science 2001, 291:118-120.

16. Feys BJ, Wiermer M, Bhat RA, Moisan LJ, Medina-Escobar N, Neu C, Cabral A, Parker JE: Arabidopsis SENESCENCE-ASSOCIATED GENE101 stabilizes and signals within an ENHANCED DISEASE SUSCEPTIBILITY1 complex in plant innate immunity. Plant Cell 2005, 17.

17. Wagner S, Stuttmann J, Rietz S, Guerois R, Brunstein E, Bautor J, Niefind K, Parker JE: Structural basis for signaling by exclusive EDS1 heteromeric complexes with SAG101 or PAD4 in plant innate immunity. Cell Host Microbe 2013, 14:619-630.

18. Daskalov A, Habenstein B, Sabate R, Berbon M, Martinez D, Chaignepain S, CoularySalin B, Hofmann K, Loquet A, Saupe SJ: Identification of a novel cell deathinducing domain reveals that fungal amyloid-controlled programmed cell death is related to necroptosis. Proc Natl Acad Sci U S A 2016, 113:2720-2725. 
19. Wu C-H, Abd-El-Haliem A, Bozkurt TO, Belhaj K, Terauchi R, Vossen JH, Kamoun S: NLR network mediates immunity to diverse plant pathogens. Proceedings of the National Academy of Sciences 2017.

20. Gabriels SH, Vossen JH, Ekengren SK, van Ooijen G, Abd-El-Haliem AM, van den Berg GC, Rainey DY, Martin GB, Takken FL, de Wit PJ, et al.: An NB-LRR protein required for HR signalling mediated by both extra- and intracellular resistance proteins. Plant J 2007, 50:14-28.

21. van der Biezen EA, Freddie CT, Kahn K, Parker JE, Jones JD: Arabidopsis RPP4 is a member of the RPP5 multigene family of TIR-NB-LRR genes and confers downy mildew resistance through multiple signalling components. Plant $J$ 2002, 29:439-451.

22. Zhang Y, Goritschnig S, Dong X, Li X: A gain-of-function mutation in a plant disease resistance gene leads to constitutive activation of downstream signal transduction pathways in suppressor of npr1-1, constitutive 1. Plant Cell 2003, 15:2636-2646.

23. Dong OX, Ao K, Xu F, Johnson KCM, Wu Y, Li L, Xia S, Liu Y, Huang Y, Rodriguez E, et al.: Individual components of paired typical NLR immune receptors are regulated by distinct E3 ligases. Nat Plants 2018, 4:699-710.

24. Ma Y, Guo H, Hu L, Martinez PP, Moschou PN, Cevik V, Ding P, Duxbury Z, Sarris PF, Jones JDG: Distinct modes of derepression of an Arabidopsis immune receptor complex by two different bacterial effectors. Proc Natl Acad Sci U S A 2018, 115:10218-10227.

25. Guo H, Ahn HK, Sklenar J, Huang J, Ma Y, Ding P, Menke FLH, Jones JDG: Phosphorylation-regulated activation of the Arabidopsis RRS1-R/RPS4 immune receptor complex reveals two distinct effector recognition mechanisms. Cell Host Microbe in press.

26. Van de Weyer AL, Monteiro F, Furzer OJ, Nishimura MT, Cevik V, Witek K, Jones JDG, Dangl JL, Weigel D, Bemm F: A Species-Wide Inventory of NLR Genes and Alleles in Arabidopsis thaliana. Cell 2019, 178:1260-1272.e1214.

27. De la Concepcion JC, Franceschetti M, MacLean D, Terauchi R, Kamoun S, Banfield $\mathrm{MJ}$ : Protein engineering expands the effector recognition profile of a rice NLR immune receptor. Elife 2019, 8.

28. Maqbool A, Saitoh H, Franceschetti M, Stevenson CEM, Uemura A, Kanzaki H, Kamoun $\mathrm{S}$, Terauchi R, Banfield MJ: Structural basis of pathogen recognition by an integrated HMA domain in a plant NLR immune receptor; 2015.

29. Longya A, Chaipanya C, Franceschetti M, Maidment JHR, Banfield MJ, Jantasuriyarat C: Gene Duplication and Mutation in the Emergence of a Novel Aggressive Allele of the AVR-Pik Effector in the Rice Blast Fungus. Mol Plant Microbe Interact 2019, 32:740-749.

30. De la Concepcion JC, Franceschetti M, Maqbool A, Saitoh H, Terauchi R, Kamoun S, Banfield MJ: Polymorphic residues in rice NLRs expand binding and response to effectors of the blast pathogen. Nat Plants 2018, 4:576-585.

31. Dangl JL, Jones JD: Plant pathogens and integrated defence responses to infection. Nature 2001, 411:826-833.

32. van der Hoorn RA, Kamoun S: From Guard to Decoy: a new model for perception of plant pathogen effectors. Plant Cell 2008, 20:2009-2017.

33. Cesari S, Bernoux M, Moncuquet P, Kroj T, Dodds PN: A novel conserved mechanism for plant NLR protein pairs: the "integrated decoy" hypothesis. Front Plant Sci 2014, 5:606.

34. Axtell MJ, Staskawicz BJ: Initiation of RPS2-specified disease resistance in Arabidopsis is coupled to the AvrRpt2-directed elimination of RIN4. Cell 2003, 112:369-377.

35. Wang J, Wang J, Hu M, Wu S, Qi J, Wang G, Han Z, Qi Y, Gao N, Wang HW, et al.: Ligand-triggered allosteric ADP release primes a plant NLR complex. Science 2019, 364. 
36. Wang J, Hu M, Wang J, Qi J, Han Z, Wang G, Qi Y, Wang HW, Zhou JM, Chai J: Reconstitution and structure of a plant NLR resistosome conferring immunity. Science 2019, 364.

37. Kim SH, Qi D, Ashfield T, Helm M, Innes RW: Using decoys to expand the recognition specificity of a plant disease resistance protein. Science 2016, 351:684-687.

38. Pottinger SE, Bak A, Margets A, Helm M, Tang L, Casteel C, Innes RW: Optimizing the PBS1 Decoy System to Confer Resistance to Potyvirus Infection in Arabidopsis and Soybean. bioRxiv 2020:2020.2001.2012.903575.

39. Adachi H, Contreras MP, Harant A, Wu CH, Derevnina L, Sakai T, Duggan C, Moratto E, Bozkurt TO, Maqbool A, et al.: An N-terminal motif in NLR immune receptors is functionally conserved across distantly related plant species. Elife 2019, 8.

40. Saur IM, Conlan BF, Rathjen JP: The N-terminal domain of the tomato immune protein Prf contains multiple homotypic and Pto kinase interaction sites. J Biol Chem 2015, 290:11258-11267.

41. Li J, Huang H, Zhu M, Huang S, Zhang W, Dinesh-Kumar SP, Tao X: A Plant Immune Receptor Adopts a Two-Step Recognition Mechanism to Enhance Viral Effector Perception. Mol Plant 2019, 12:248-262.

42. Hofmann K: The Evolutionary Origins of Programmed Cell Death Signaling. Cold Spring Harb Perspect Biol 2019.

43. Mahdi L, Huang M, Zhang X, Nakano RT, Kopp LB, Saur IML, Jacob F, Kovacova V, Lapin D, Parker JE, et al.: Plant mixed lineage kinase domain-like proteins limit biotrophic pathogen growth. bioRxiv 2019:681015.

44. Barragan CA, Wu R, Kim ST, Xi W, Habring A, Hagmann J, Van de Weyer AL, Zaidem $\mathrm{M}, \mathrm{Ho} W W H$, Wang $\mathrm{G}$, et al.: RPW8/HR repeats control NLR activation in Arabidopsis thaliana. PLoS Genet 2019, 15:e1008313.

45. Castel B, Wu Y, Xiao S, Jones JDG: An rpw8 quadruple mutant of Arabidopsis Col-0 is partially compromised in bacterial and fungal resistance. bioRxiv 2019:839308.

46. Shao Z-Q, Xue J-Y, Wu P, Zhang Y-M, Wu Y, Hang Y-Y, Wang B, Chen J-Q: LargeScale Analyses of Angiosperm Nucleotide-Binding Site-Leucine-Rich Repeat Genes Reveal Three Anciently Diverged Classes with Distinct Evolutionary Patterns. Plant Physiology 2016, 170:2095-2109.

47. Wu Z, Li M, Dong OX, Xia S, Liang W, Bao Y, Wasteneys G, Li X: Differential regulation of TNL-mediated immune signaling by redundant helper CNLs. New Phytol 2019, 222:938-953.

48. Andolfo G, Villano C, Errico A, Frusciante L, Carputo D, Aversano R, Ercolano MR: Inferring RPW8-NLRs's evolution patterns in seed plants: case study in Vitis vinifera. Planta 2019, 251:32.

49. Baggs EL, Thanki AS, O'Grady R, Schudoma C, Haerty W, Krasileva KV: Convergent loss of an EDS1/PAD4 signalling pathway in several plant lineages predicts new components of plant immunity and drought response. bioRxiv 2019:572560.

50. Lapin D, Kovacova V, Sun X, Dongus JA, Bhandari DD, von Born P, Bautor J, Guarneri N, Rzemieniewski J, Stuttmann J, et al.: A coevolved EDS1-SAG101-NRG1 module mediates cell death signaling by TIR-domain immune receptors. Plant Cell 2019.

51. Saile SC, Jacob P, Castel D, Jubic LM, Gonzalez IS, Bäcker M, Jones JDG, Dangl JL, El Kasmi F: Plant helper NLRs act similar to canonical CNLs to mediate and sustain effector-triggered immunity downstream of sensor NLR activation. BioRxiv submitted.

52. Qi T, Seong K, Thomazella DPT, Kim JR, Pham J, Seo E, Cho MJ, Schultink A, Staskawicz BJ: NRG1 functions downstream of EDS1 to regulate TIR-NLRmediated plant immunity in Nicotiana benthamiana. Proc Natl Acad Sci U S A 2018, 115:E10979-e10987. 
53. Gantner J, Ordon J, Kretschmer C, Guerois R, Stuttmann J: An EDS1-SAG101 Complex Is Essential for TNL-Mediated Immunity in Nicotiana benthamiana. Plant Cell 2019, 31:2456-2474.

54. Castel B, Ngou PM, Cevik V, Redkar A, Kim DS, Yang Y, Ding P, Jones JDG: Diverse NLR immune receptors activate defence via the RPW8-NLR NRG1. New Phytol 2019, 222:966-980.

55. Brendolise C, Martinez-Sanchez M, Morel A, Chen R, Dinis R, Deroles S, Peeters N, Rikkerink EHA, Montefiori M: NRG1-mediated recognition of HopQ1 reveals a link between PAMP and Effector-triggered Immunity. bioRxiv 2018.

56. Schultink A, Qi T, Lee A, Steinbrenner AD, Staskawicz B: Roq1 mediates recognition of the Xanthomonas and Pseudomonas effector proteins XopQ and HopQ1. Plant J 2017, 92:787-795.

57. Horsefield S, Burdett H, Zhang X, Manik MK, Shi Y, Chen J, Qi T, Gilley J, Lai JS, Rank $\mathrm{MX}$, et al.: NAD(+) cleavage activity by animal and plant TIR domains in cell death pathways. Science 2019, 365:793-799.

58. Wan L, Essuman K, Anderson RG, Sasaki Y, Monteiro F, Chung EH, Osborne Nishimura E, DiAntonio A, Milbrandt J, Dangl JL, et al.: TIR domains of plant immune receptors are $\mathrm{NAD}(+)$-cleaving enzymes that promote cell death. Science 2019, 365:799-803.

59. Duxbury Z, Wang S, MacKenzie Cl, Tenthorey JL, Zhang X, Un Huh S, Hu L, Hill L, Ngou PK, Ding P, et al.: Induced proximity of a TIR signaling domain on a plantmammalian 3 NLR chimera activates defense in plants. PNAS submitted. 
COI statement

The authors declare no conflicts of interest 